\title{
A MULTIDISCIPLINARY APPROACH FOR THE REHABILITATION OF A PATIENT WITH CHONDROSARCOMA: PROSTHETICALLY-DRIVEN DIGITAL WORKFLOW FOR MAXILLARY RECONSTRUCTION AND IMPLANT TREATMENT
}

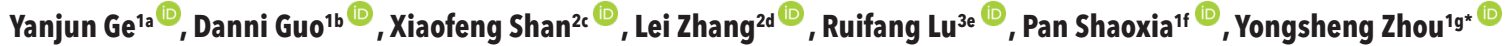 \\ 'Department of Prosthodontics, Peking University School and Hospital of Stomatology \& National Center of Stomatology \& National Clinical \\ Research Center for Oral Diseases \& National Engineering Laboratory for Digital and Material Technology of Stomatology \& Beijing Key \\ Laboratory of Digital Stomatology \& Research Center of Engineering and Technology for Computerized Dentistry Ministry of Health \& NMPA \\ Key Laboratory for Dental Materials, Beijing 100081, China \\ ${ }^{2}$ Department of Oral-Maxillofacial Surgery, Peking University School and Hospital of Stomatology \& National Center of Stomatology \& \\ National Clinical Research Center for Oral Diseases \& National Engineering Laboratory for Digital and Material Technology of Stomatology, \\ Beijing 100081, China \\ ${ }^{3}$ Department of Periodontology, Peking University School and Hospital of Stomatology \& National Center of Stomatology \& National Clinical \\ Research Center for Oral Diseases \& National Engineering Laboratory for Digital and Material Technology of Stomatology \& Beijing Key \\ Laboratory of Digital Stomatology \& Research Center of Engineering and Technology for Computerized Dentistry Ministry of Health \& NMPA \\ Key Laboratory for Dental Materials, Beijing 100081, China
}

aDDS, PhD; e-mail:yanjun ge@163.com; ORCIDiD: https://orcid.org/0000-0003-2853-8657
'DDS; e-mail: gdnsunshine@163.com; ORCIDiD: https://orcid.org/0000-0001-8190-0972
'DDS, PhD; e-mail: kqsxf@263.net; ORCIDiD: https://orcid.org/0000-0002-2126-8052
dDDS, PhD; e-mail:zhlei doctor@sina.com; ORCIDiD: https://orcid.org/0000-0001-8251-6032
e DDS, PhD; e-mail: kgrflu@bjmu.edu.cn; ORCIDiD: https://orcid.org/0000-0002-9581-508X
'DDS, PhD; e-mail: panshaoxia@vip.163.com; ORCID: https://orcid.org/0000-0002-3808-9499
'DDS, PhD, Professor and Head; e-mail: kqzhouysh@hsc.pku.edu.cn; ORCID: $\underline{\text { https://orcid.org/0000-0002-4332-0878 }}$

d) https://doi.org/10.25241/stomaeduj.2021.8(3).art.7

ABSTRACT

Aim To describe a comprehensive digital therapy oriented towards the final restoration for treating an oral maxillofacial defect caused by maxillary chondrosarcoma.

Summary The prosthetically-driven multidisciplinary approach was applied to achieve perfectly functionalaesthetic reconstruction for a male patient with maxillary chondrosarcoma. The complete tumor resection was ensured by the design of virtual osteotomy and surgical guide plate. A reverse engineering technique was used to reconstruct the bone defect in the maxillary aesthetic area, which offered reference for a threedimensional printing guide plate to shape and fix the free vascularized iliac bone flap. On the solid basis of previous treatment, the implant placement was performed under the guidance of the prosthetic-driven implant plate. Vestibular extension and tissue graft were performed to increase keratinized gingiva width to improve implant-supported fixed prosthesis effect.

Key learning points 1. A multidisciplinary approach including maxillofacial surgery, prosthodontic and periodontal treatment can provide better esthetic and functional results for complex rehabilitation of a patient with oral maxillofacial defect. 2. Predictability of maxillary reconstruction and implant restoration can be increased with prosthetic-driven treatment plan. 3. Applying preoperative virtual design and personalized guide plate is beneficial to achieve an ideal outline of reconstructed upper jaw. 4. Obtaining comprehensive aesthetic parameters of the expected restoration is one of the key principles of upper anterior teeth rehabilitation. 5. Digital technology provides an opportunity for consistency between the primary treatment design and the final restoration outcome.

\section{KEYWORDS}

Digital; Surgical guide; Implant supported restoration; Oral-maxillofacial defect; Multidisciplinary approach

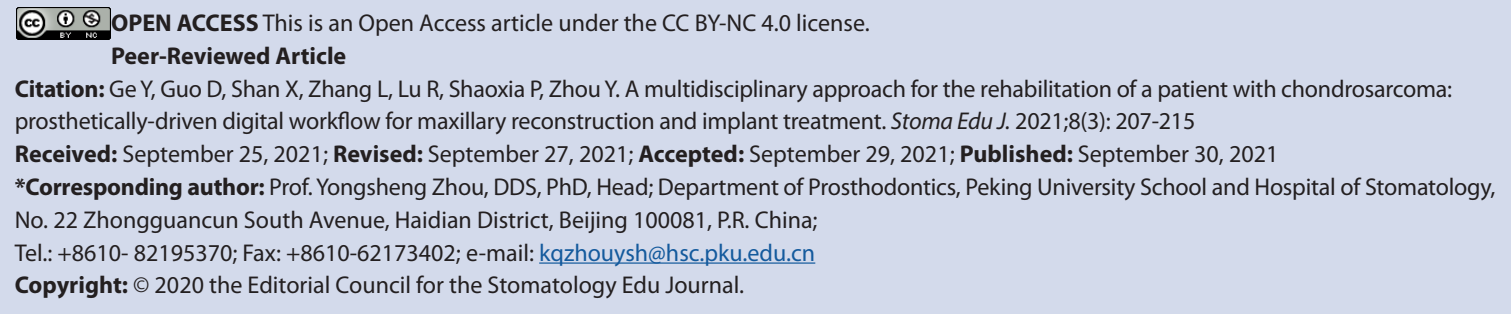




\section{INTRODUCTION}

Tumors, trauma or congenital factors mostly lead to an oral-maxillofacial defect. The maxillary defect, especially in the anterior teeth area, has a serious impact on patients from both physiological and psychological aspects, including bite, pronunciation and aesthetic functions $[1,2]$. Therefore, as a complex functional and aesthetic reconstruction, the multidisciplinary approach is urgently needed to make a comprehensive diagnosis and optimal treatment plan, which may consist in periodontal, prosthodontic, maxillofacial and implant treatment [3].

Conventional rehabilitation workflow for patients with oral cancer completes the following steps in sequence [4]. Surgery is performed to remove the affected area. The bone defect is reconstructed with a bone flap or prosthesis. When feasible, the implant and fixed restoration are used to improve the effect. Obviously, there are disadvantages of the conventional workflow to be optimized. Discontinuous procedures are difficult to ensure the treatment consistency. The diagnosis and treatment standards of different specialties are diversified and rely heavily on their experience. In summary, the complex treatment process reduces the predictability of the rehabilitation [5].

The development of digital technology provides a variety of means for the optimization of the oralmaxillofacial defect treatment, such as virtual surgery, personalized model made by rapid prototyping technique, static plate or dynamic navigation-guided surgery [6]. The application of the novel digital technology can achieve higher time efficiency and better quality of outcomes for prosthetic treatment [7]. The implant guide plate significantly reduces the error of implant surgery [8]. Precious registration of multi-source data can realize virtual aesthetic design before surgery. Most of the current scientific evidence of applying digital technique for oral-maxillofacial rehabilitation focuses on mandible reconstruction and shaping of vascularized fibular flap [5,9-12]. Literature on maxillary reconstruction in aesthetic high-risk area rehabilitated with a free vascularized iliac bone flap assisted by digital technique was rare.

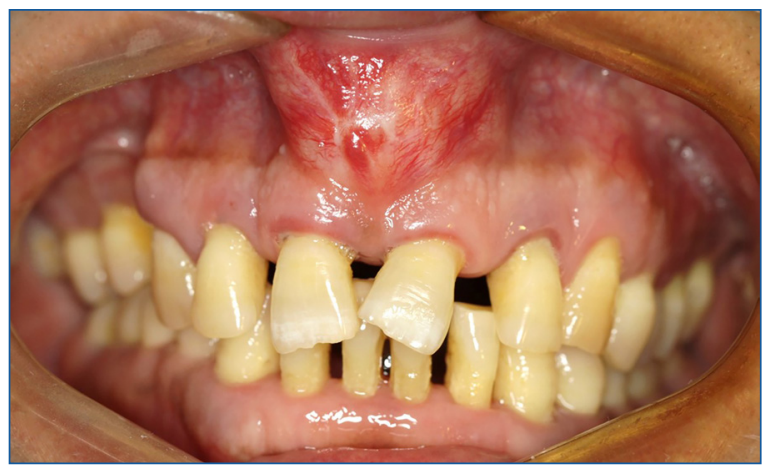

IFigure 1. A maxillary tumor with a diameter of about $3 \mathrm{~cm}$ and smooth surface on the labial side of the upper anterior teeth.
In this article, a maxillary chondrosarcoma case is reported to propose a multidisciplinary approach and prosthetically-driven digital workflow for the oral-maxillofacial rehabilitation.

\section{CASE PRESENTATION}

A 46-year-old male patient required examination and treatment with the chief complaint of a mass on the front of the maxillary and accompanied by loose upper anterior teeth for several months, and he had no relevant medical history. The general examination showed no abnormalities. The patient visited the Department of Prosthodontics, Peking University School and Hospital of Stomatology in February 2018.

There was a tough mass with a diameter of about $3 \mathrm{~cm}$ and smooth surface on the labial side of the maxilla central and lateral incisors (Fig. 1). The vestibular sulcus was swollen without bleeding and tenderness. Intraoral examination revealed tooth space among upper anterior teeth, which were I degree loose and drifted towards the mesial direction. Deep overjet and overbite were shown on anterior teeth. The first and second right maxilla premolars were missing. The relative position of the upper and lower jaws was basically normal. This patient had lots of dental calculus and poor oral hygiene. The contour of the nasal base and upper lip was obviously raised, the skin color of the lesion looking normal. There were no abnormalities in the region of the neurological lymph nodes and bilateral temporomandibular joint.

Cone beam computed tomography (CBCT) showed one circular area in the maxilla with reduced bone density and unclear boundaries closely adhering to the anterior teeth. The range of this lesion was about $5 \mathrm{~cm} \times 4 \mathrm{~cm} \times 3 \mathrm{~cm}$, in which spot and flakelike calcification were visible. The radiographic film revealed neither root canal treatment nor peri-apical resorption of upper anterior teeth (Fig. 2).

According to the clinical and radiographic examination, multidisciplinary experts consulted and gave a comprehensive diagnosis and sequential treatment plan. Firstly, the systematic periodontal treatment was performed by the periodontist.

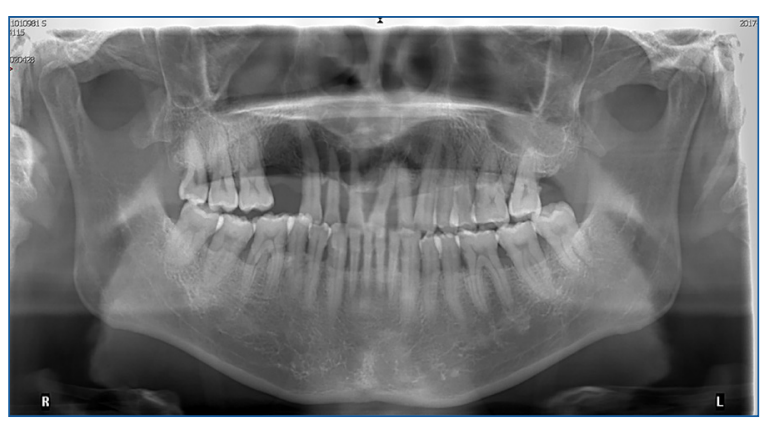

Figure 2. Pre-operative panoramic radiograph indicating the maxilla with reduced bone density and unclear boundaries lesion close to the anterior teeth. Neither root canal treatment nor peri-apical resorption of upper anterior teeth were noted. 
The oral and maxillofacial surgeons would remove the maxillary tumor and reconstruct the maxilla for implant restoration. Free gingival grafts would be performed where required. Subsequently, implant-supported fixed partial denture would be accomplished.

The current condition, treatment plan, cost and prognosis were communicated in detail to the patient. Signed informed consent, with the aid of digital technique, virtual surgical planning (VSP) and rapid prototyping technology (RP) were applied for maxillary chondrosarcoma resection and reconstruction. The free vascularized iliac bone graft surgery was performed. The CT dataset of maxillofacial and iliac were used to simulate the osteotomy operation and construct a three-dimensional maxillary defect model through reverse engineering software. Based on digital design, surgical guide plates and personalized models were made to assist in the osteotomy operation and flap fixation (Fig. 3). A full-thickness flap was raised through intraoral incision under general anesthesia. The toothsupported maxillary surgical guide was completed in place. According to the preoperative design, the maxillary lesion was excised (Fig. 4). Taking pathology analysis and examination together, the results confirmed the diagnosis of well-differentiated chondrosarcoma. According to the pathological results of frozen sections, there wereas no tumor cells at the margin. The free vascularized iliac bone flap was prepared and shaped under the guidance of the personalized plate and maxillary model. The bone flap was adequately positioned at the maxillary defect area and firmly fixed by titanium nails and plate. A vascular anastomosis was completed under the microscope for the successful reconstruction (Fig. 5). One week, three months and twelve months after surgery, a clinical examination was performed at each follow-up visit. Panoramic radiograph and CBCT showed no recurrence (Fig. 6).

After healing, plaster models were made from primary impressions. A characteristic occlusal rim was made to record the maxillo-mandibular relationship and key aesthetic parameters with radiopaque material. The Digital Imaging and Communications in Medicine (DICOM) data from CBCT and model scanning data were registered accurately. Based on the aesthetic principle, the expected restoration and implant treatment were virtually designed (Fig. 7). An implant surgical guide supported by natural teeth and lateral retention nails was made through the RP technique. A full-thickness flap was raised under local anesthesia (Primacaine adrenaline 1:100,000, Dentaires Pierre Rolland), and four implants (15,13 NobelActive ${ }^{\circledR}$ RP, 11,21 NobelActive ${ }^{\oplus}$ NP, Institut Nobel Biocare AG) were inserted with the guidance of the template (Fig. 8). Before suturing, the healing screws were connected to the implant. Ten days after surgery, the sutures were removed. The examination revealed a favorable healing process of the implant and soft tissue.

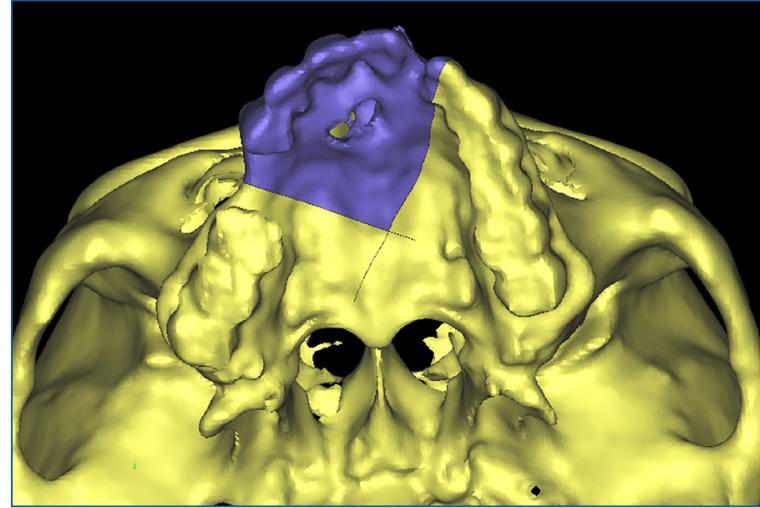

IFigure 3. (a)

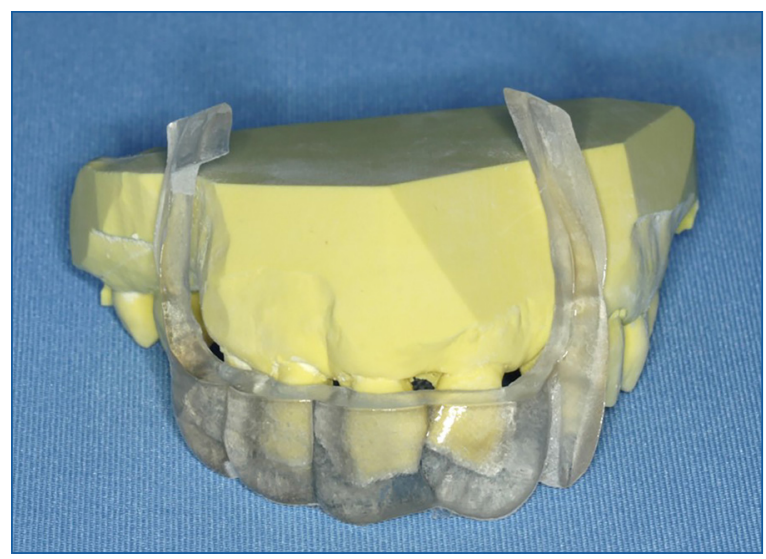

IFigure 3.(b)

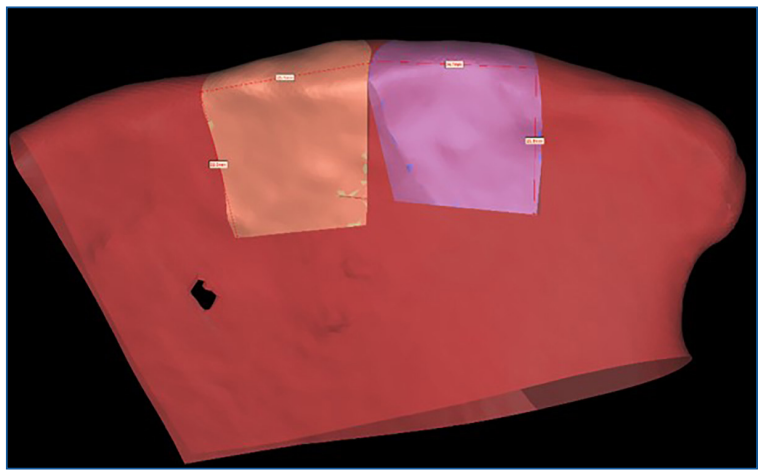

IFigure 3. (c)

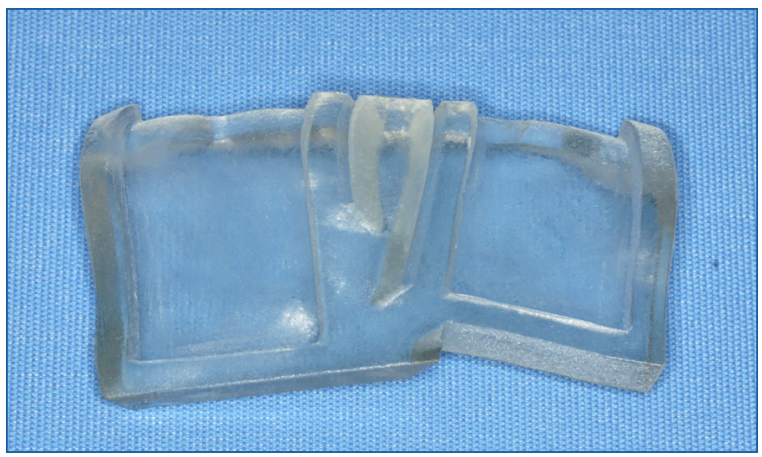

IFigure 3.(d)

I Figure 3. Virtual surgical planning (VSP) and rapid prototyping technology (RP) applying for maxillary chondrosarcoma resection and reconstruction. (a) Virtual osteotomy operation was performed using digital technique. (b) 3D printed surgical plate for radical resection of maxillary chondrosarcoma. (c) The iliac CT data were used to simulate maxillary defect reconstruction. (d) A guide plate for free vascularized iliac bone graft surgery. 


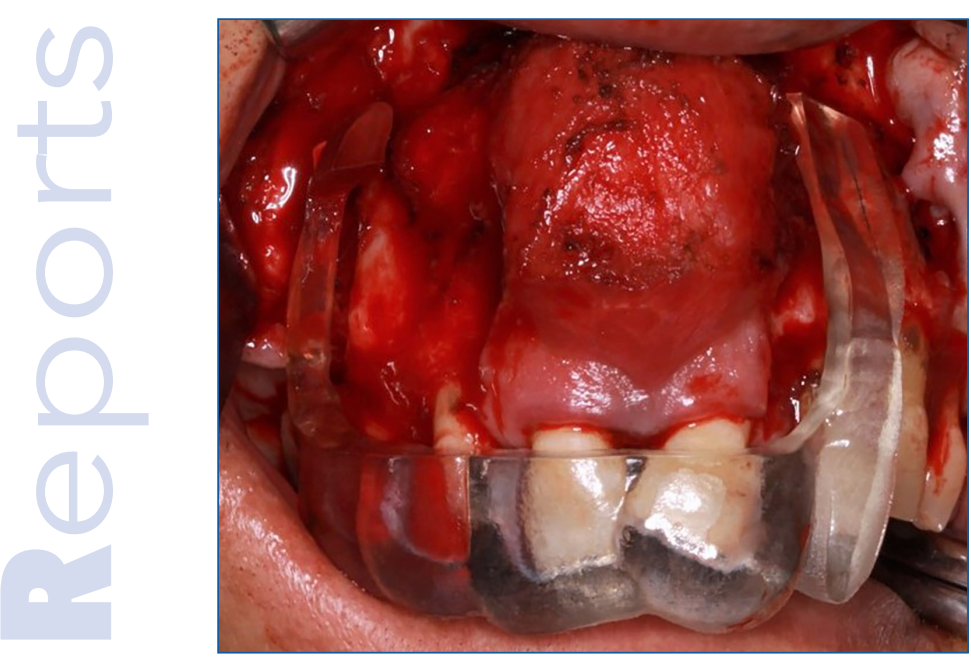

IFigure 4. (a)

I Figure 4. Resection of maxillary chondrosarcoma with the guidance of tooth-supported surgical guide. (a) The surgical guide plate was in place. (b) The maxillary lesion was excised as pre-operative design. (c) The maxillary defect after maxillofacial surgery.

I Figure 5. Maxillary reconstruction with vascularized iliac bone flap graft surgery. (a) Preparation of free vascularized iliac bone flap. (b) Shaping and fixation of flap with the personalized 3D printed model as reference. (c) The bone flap was adequately positioned at the maxillary defect area and firmly fixed. (d) Completion of vascular anastomosis.

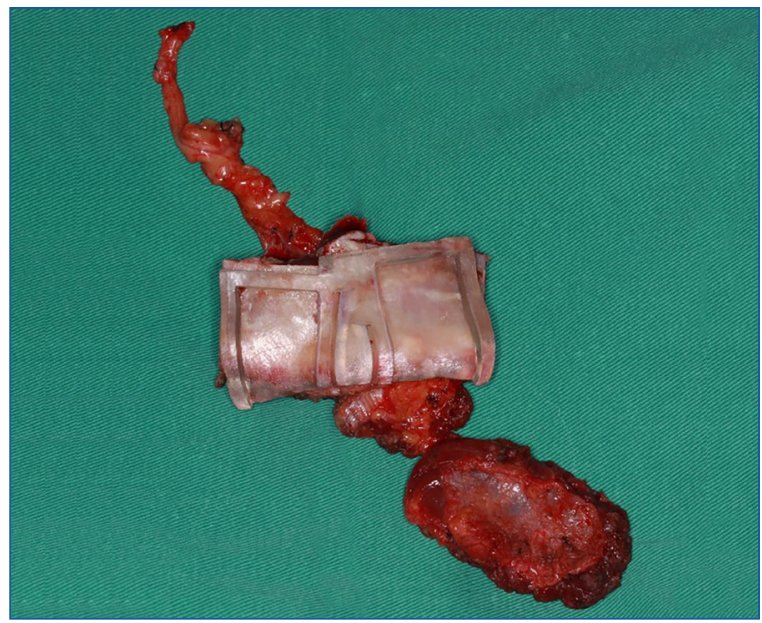

IFigure 5.(a)

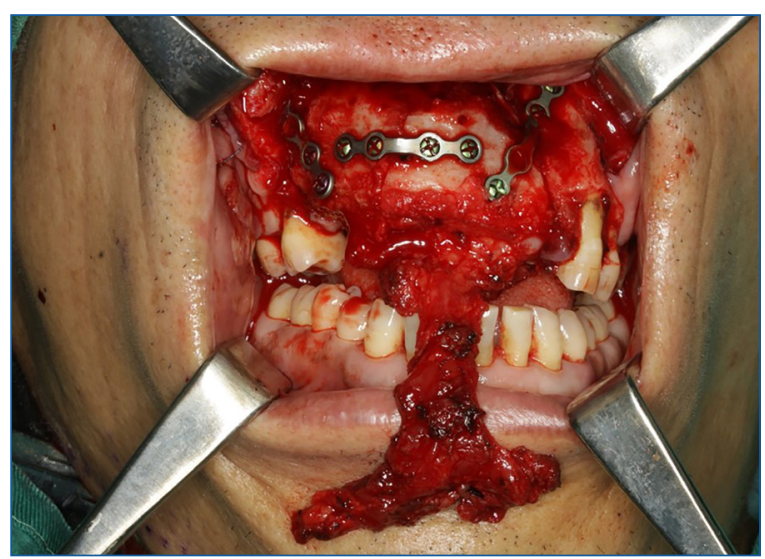

IFigure 5. (c)

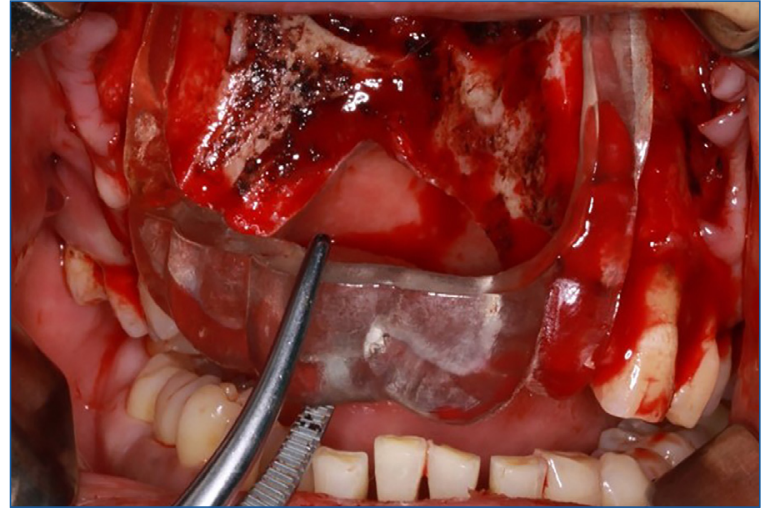

IFigure 4. (b)

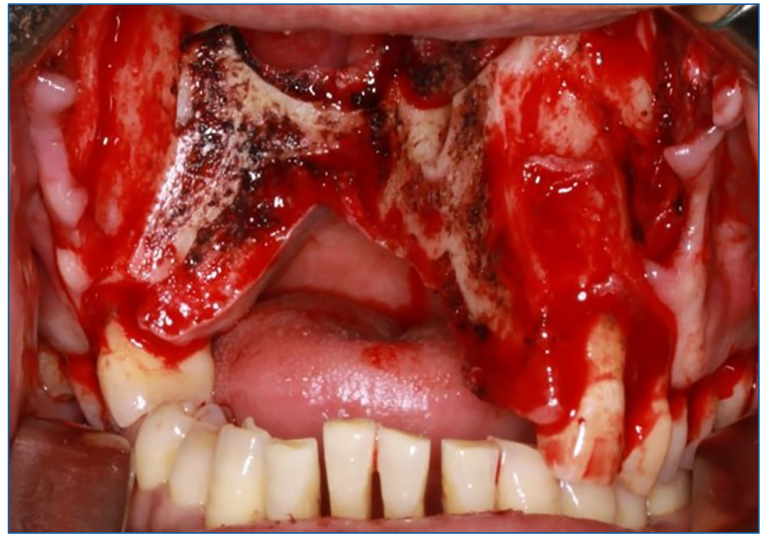

IFigure 4. (c)

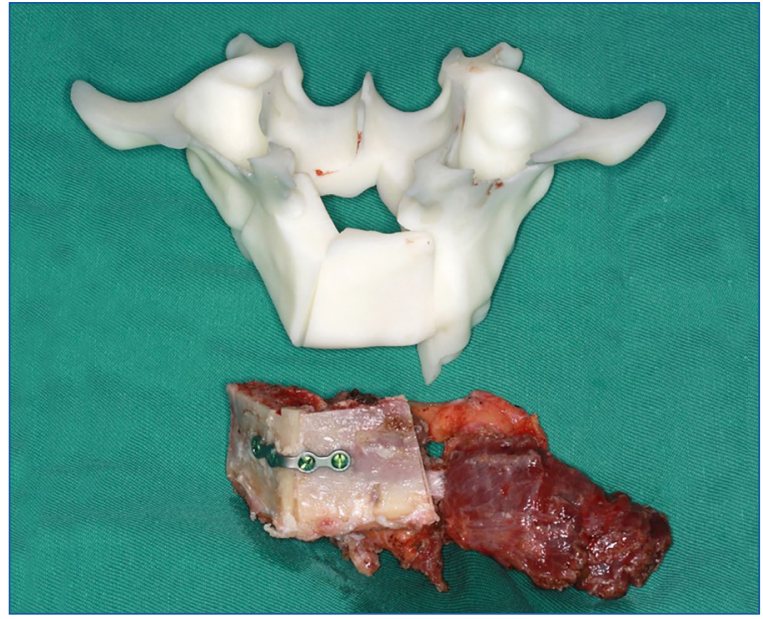

IFigure 5.(b)

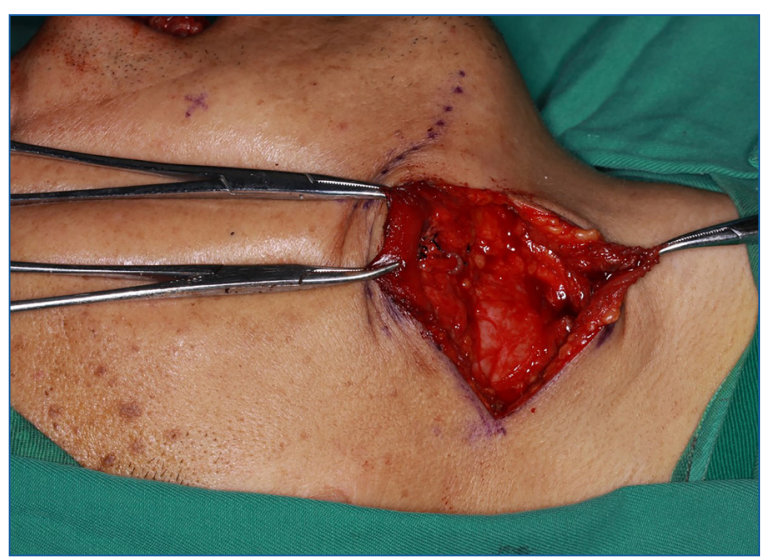

IFigure 5.(d) 


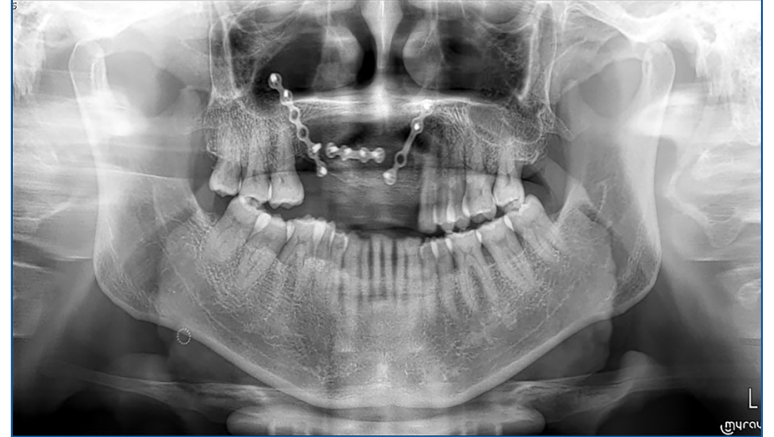

I Figure 6. Panoramic radiograph showed adequate bone for implant placement.

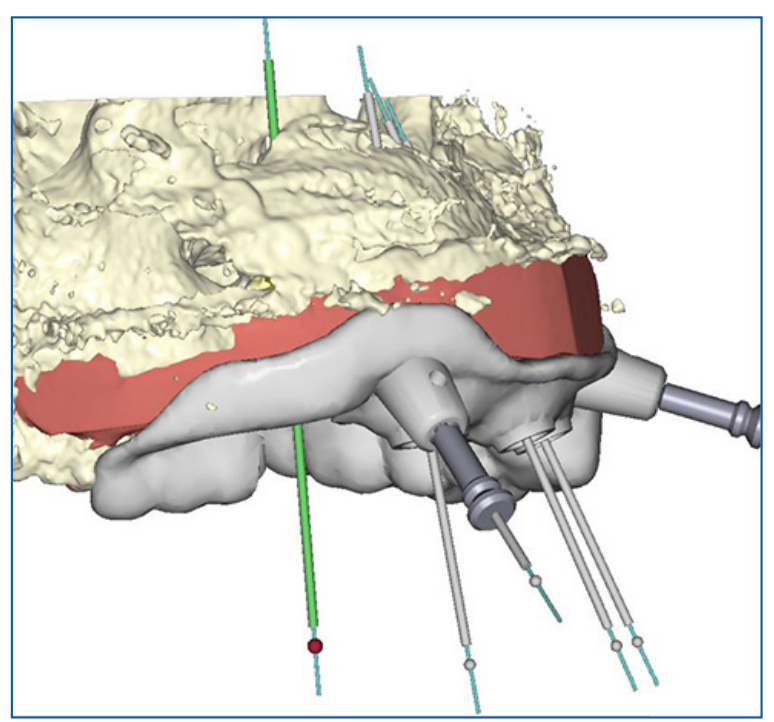

IFigure 7.(a)

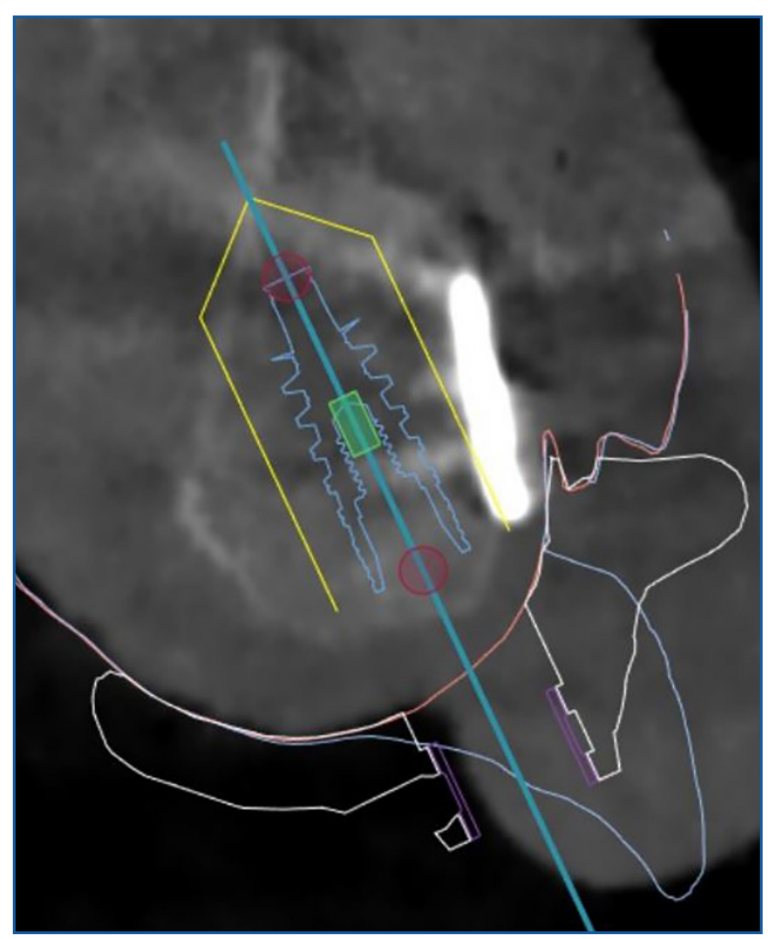

IFigure 7.(b)

I Figure 7. A prosthetically-driven virtual design for implant placement. (a) Registration of multi-source data. (b) Implant type and position were virtually designed according to the optimal restoration.

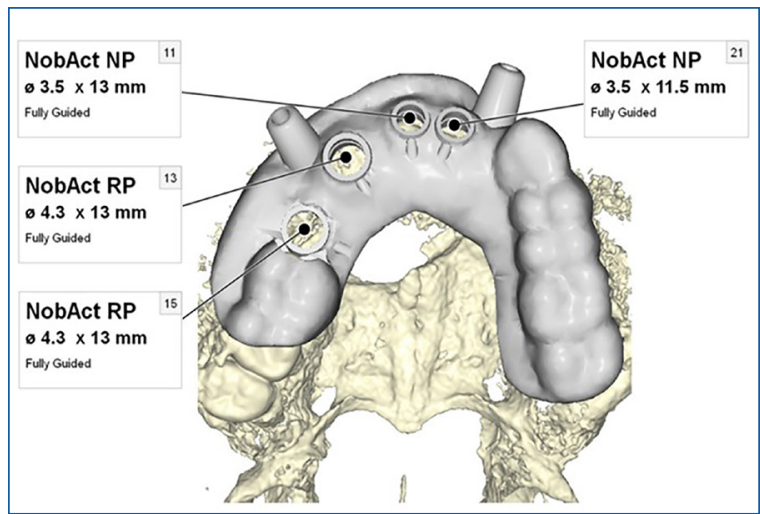

IFigure 8. (a)

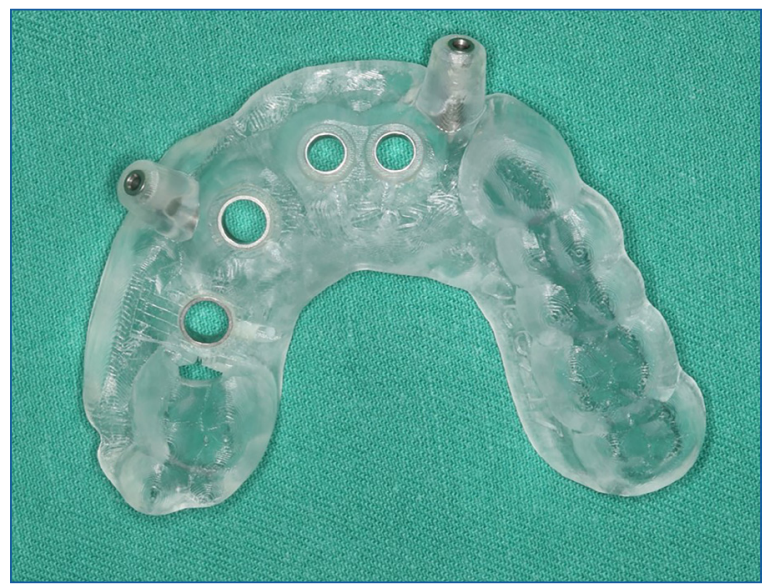

IFigure 8. (b)

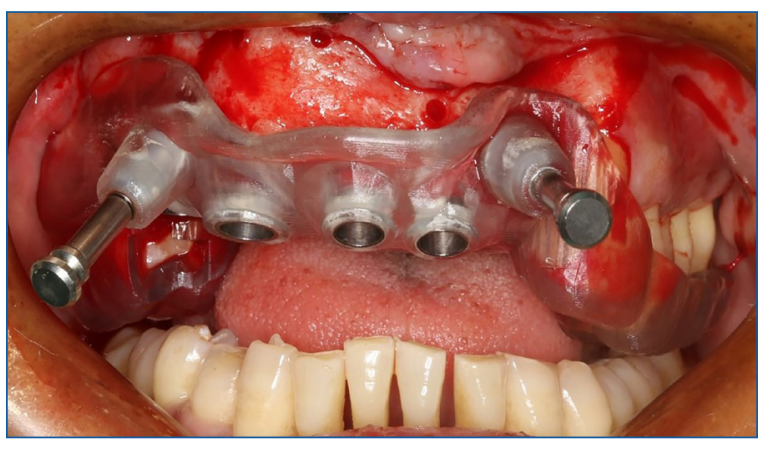

IFigure 8. (c)

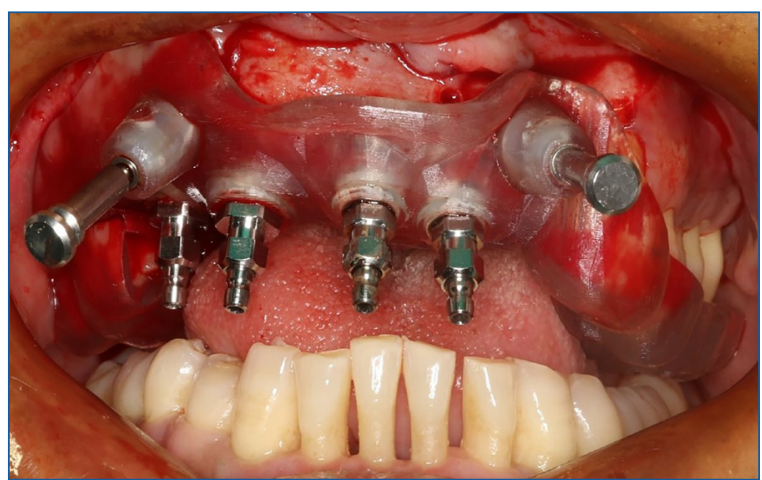

IFigure 8. (d)

I Figure 8. A restoration-oriented digital workflow for implant surgery. (a) Implant treatment was virtually designed using precious registered dataset. (b) Implant surgical guide was made by rapid prototyping technology. (c) The implant guide plate was stabilized through transverse retention nails and residual teeth. (d) Optimal implant placement under the guidance of the plate. 


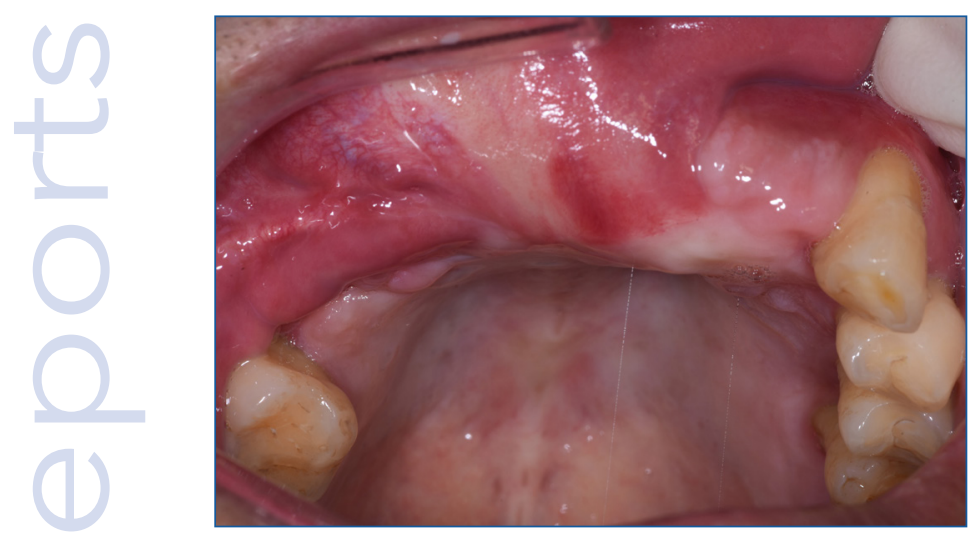

IFigure 9. (a)

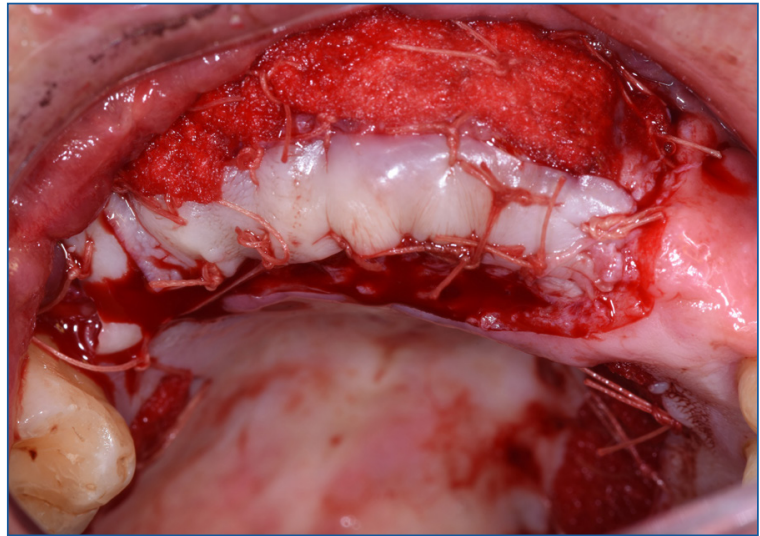

IFigure 9. (b)

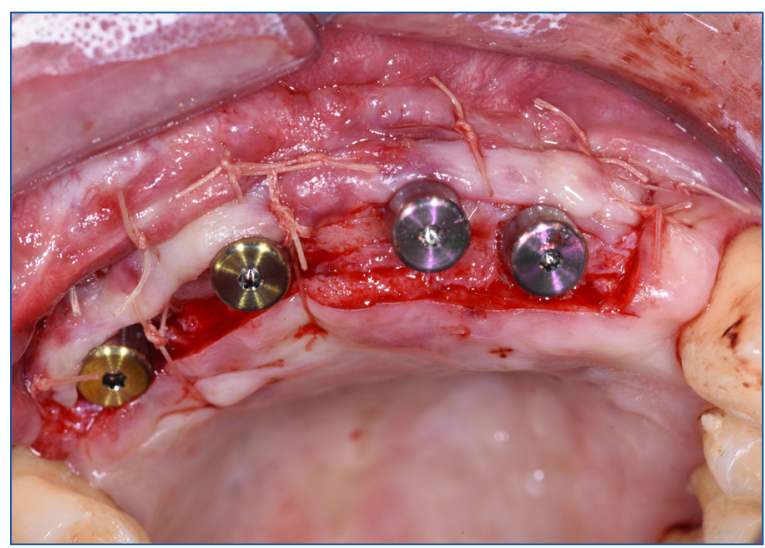

IFigure 9. (c)

I Figure 9. Tissue graft to increase width of the keratinized gingiva. (a) A deficiency of the keratinized gingiva on the buccal side of the right upper jaw. (b) Performance of vestibular extension and tissue graft. (c) The second-stage operation and apically repositioned flap surgery.

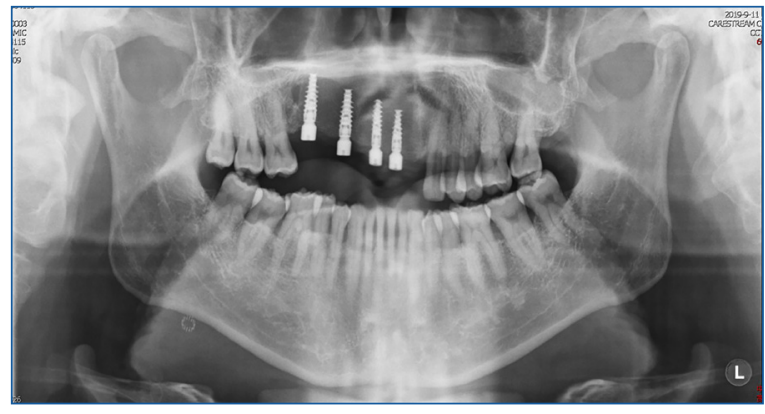

IFigure 10. Panoramic radiograph after osseointegration of implants.

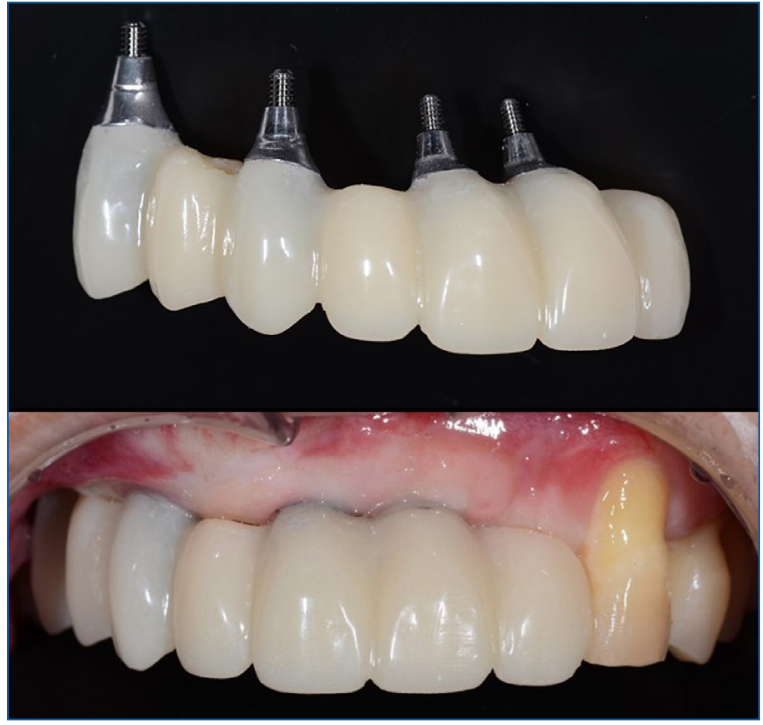

Figure 11. Temporary resin restoration milled with a 5-axis CNC machine.

Four months after surgery, the implants showed good osseointegration. Further intraoral examination showed less keratinized gingiva on the buccal side of the right upper jaw. Vestibular extension and tissue graft were performed to increase keratinized gingiva width. During the second-stage operation, the $4 \mathrm{~mm}$ buccal keratinized gingival was retained through apically repositioned flap surgery (Fig. 9). The screws were unscrewed, and the healing abutments were connected to the implants (Fig. 10).

Communicating with the patient, the final restoration was screw-retained zirconia base with labial porcelain fixed bridge. For the upper jaw, an open-tray splint implant impression was taken with a polyether material (Impregum Penta, 3M ESPE GmbH). A conventional impression of the opposing jaw was taken with alginate material (Alginoplast, Heraeus Kulzer GmbH). Temporary resin restoration was milled with a 5 -axis CNC machine. Trying in the temporary restoration, passive fit and appropriate upper lip fullness were achieved (Fig. 11). Referred to the pupil line and smile line, minus adjustment of the denture was performed on the restoration to obtain coordinated midline and incisal curve. After three months, the patient did not express discomfort. The maxillo-mandibular relationship was redetermined and transferred to the articulator by facebow. The final zirconium restoration was made with the reference of the temporary restoration. At delivery, the interproximal contact points were assessed for a strong contact using dental floss. Occlusal contact points were checked for light occlusal contacts without lateral occlusal disturbance (Arti冈Fol shimstock foil, Dr. Jean Bausch $\mathrm{GmbH} \&$ Co.). With a small amount of modified occlusal contact, the patient showed high satisfaction for the effect and comfort (Fig. 12). The panoramic radiograph showed good marginal adaptation of final restoration (Fig. 13). Twelve months after delivery, the follow-up examination revealed restoration in good condition. Oral hygiene maintenance and regular recheck were instructed (Fig. 14). 


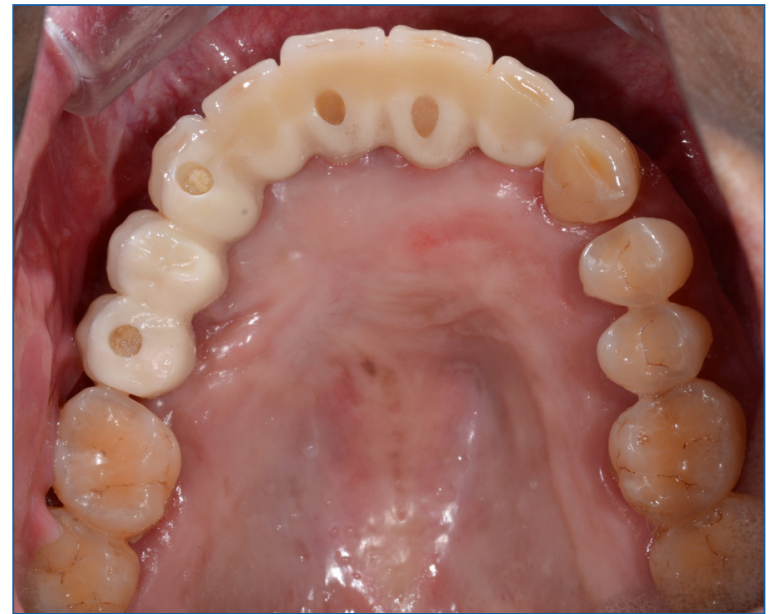

IFigure 12. (a)

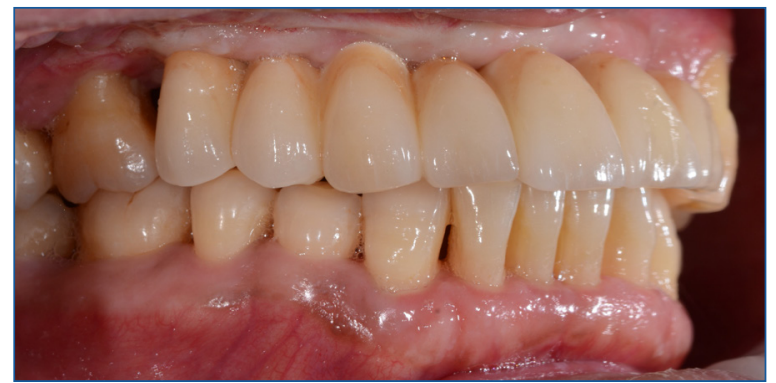

Figure 12.(b)

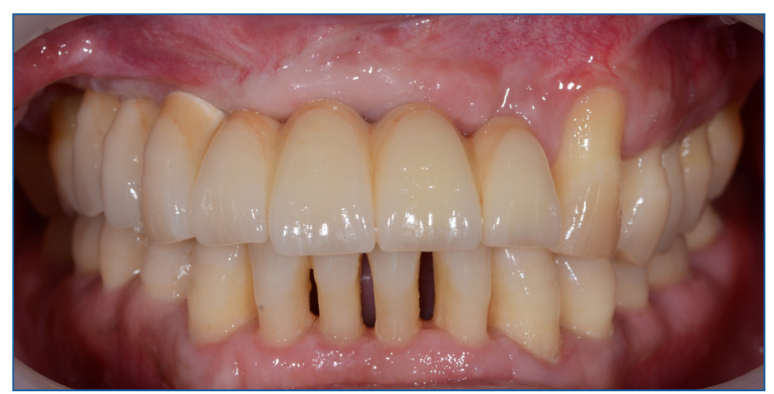

IFigure 12. (c)

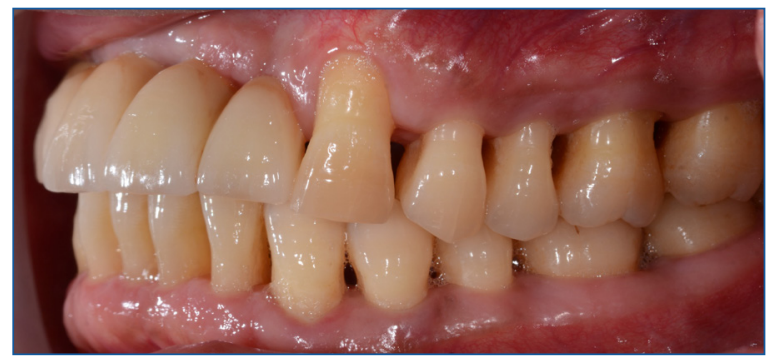

Figure 12.(d)

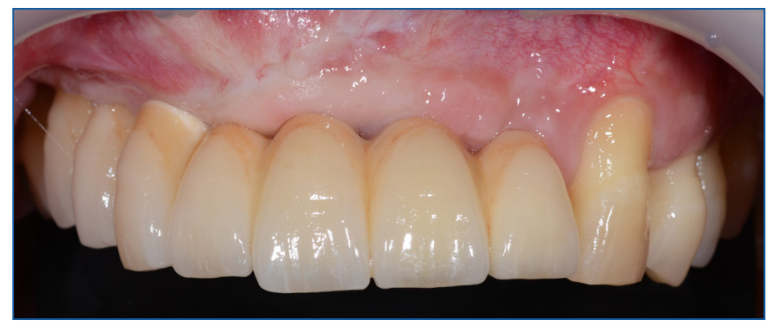

IFigure 12. (e)

I Figure 12. Perfect functional-aesthetic reconstruction with final implant supported fixed partial denture. (a) Occlusal view. (b) Centric occlusion-right. (c) Centric occlusion-left. (d) The color and contour of the restoration are consistent with adjacent teeth. (e) The gingival end of the bridge is in harmony with the soft tissue.

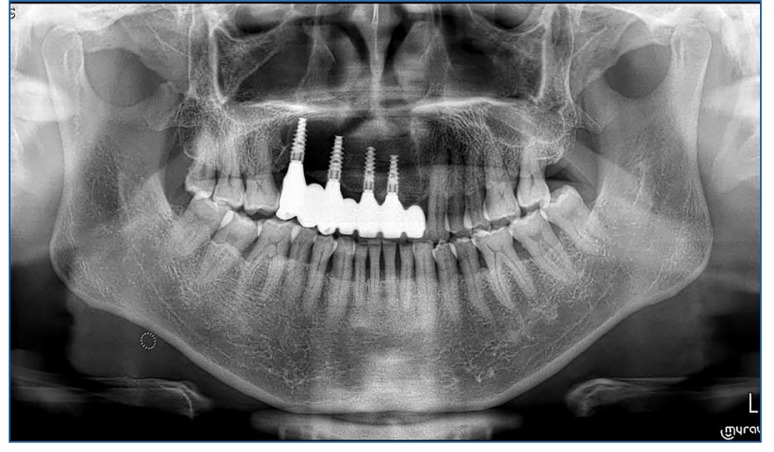

IFigure 13. Panoramic radiograph after final restoration showed passive fit.

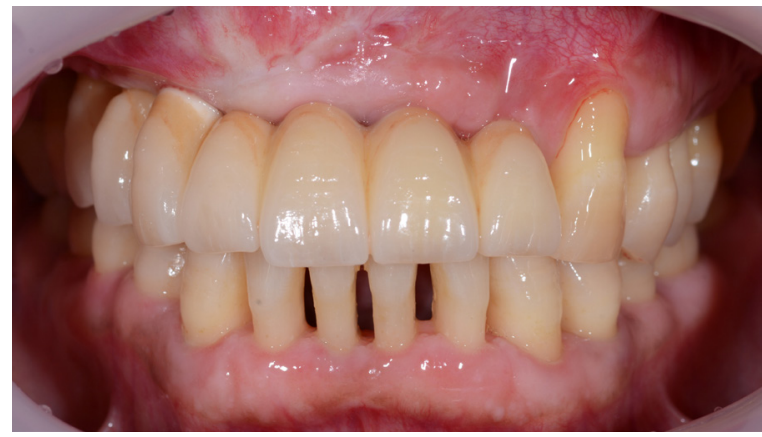

IFigure 14. (a)

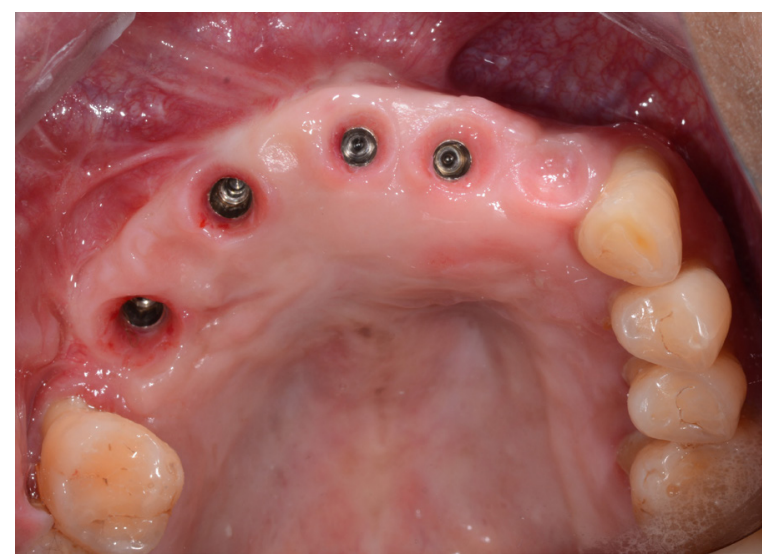

Figure 14.(b)

I Figure 14. Twelve months after delivery. (a) Restoration in good condition (b) Well maintained oral hygiene.

\section{DISCUSSION}

In this case, the patient was diagnosed with maxillary chondrosarcoma, which is a rare malignant neoplasm [13]. Chondrosarcoma(CHS) usually occurs in the pelvis, chest wall, and scapula. Maxillary chondrosarcoma is more common in men, average age range from 35 to 45 years old [14]. Maxillary chondrosarcoma may originate from the embryonic cartilage precursors of turbinate and nasal septum. According to the characteristics of the cells under the microscope, this patient is pathologically diagnosed as highly differentiated chondrosarcoma, classified as Grade I. The 5-year survival rate for patients with grade I is $89 \%$, according to WHO data $[13,15]$. Therefore, it is necessary to provide complete treatment and a rehabilitation plan for this patient to improve his survival life quality. 
Prosthetic-driven multidisciplinary approach is an important concept for maxillofacial defect rehabilitation [5]. Radical resection of the affected area of maxillary chondrosarcoma is the sole way to achieve an optimal outcome. However, common hard and soft tissue defects after resection always make it too difficult to complete optical restoration. In this case, under the guidance of final implant supported fixed restoration, the key parameters were formulated to shape the iliac bone flap, and to restore the contour and bone mass of the maxillary defect area. Based on the solid foundation, ideal implant surgery can be performed as the established design with the aid of a digital surgical guide plate. At the same time, a plan to increase the width of keratinized gingiva with palatal mucosal transplantation is made when necessary [19]. Prosthetic-driven treatment can improve the predictability of rehabilitation effect, the consistency of multidisciplinary treatment standards and the effectiveness of communication with patients.

The application of computer aided surgery (CAS) has significantly improved the accuracy and safety of the jaw reconstruction. Based on different bone defects, guided bone regeneration (GBR), onlay bone graft, distraction osteogenesis, or vascularized bone flap graft can be selected for reconstruction [17-19]. As agreed with the patient, the vascularized iliac bone flap was chosen to reconstruct his maxilla. Three common digital techniques are navigation surgery, guide plate, and a combination of navigation and plate, which can be selected according to the purpose of the operation and scope of the defect. In this case, using a static guide plate as appropriate auxiliary method to accurately guide osteotomy and fixation of the flap, consists three key steps: obtaining registered data pre-operation, virtual surgical planning, application of rapid prototype.
Implant restoration can effectively improve the rehabilitation outcome of patients with maxillofacial defects. It is reported that the cumulative survival rate of implants placed in bone flaps in jaw rehabilitation can reach $93.2 \%$ [20]. It should be noted that the long-term good prognosis of implant restoration is closely related to the dentist's treatment as well as the patient's maintenance. A reasonable preoperative design ensures a good self-cleaning for the patient. Hygiene maintenance and regular review effectively reduce complications. The limitation of this case report is that the temporary denture does not use digital preoperative design. The application of the fully digital workflow can further ensure the guiding role of the final restoration. The conventional workflow used in this case report is a time-saving and mature method. The long-term repair effect needs to be further tracked.

\section{CONCLUSION}

In this case, a new concept of multidisciplinary diagnosis and treatment approach is used to complete the maxillary reconstruction and implant supported fixed restoration for the maxillary chondrosarcoma patient. The prosthetic-driven workflow can achieve the consistency of complex treatment standards and improve the predictability of the restoration. The accuracy and efficiency of the treatment are improved with digital technology.

\section{CONFLICT OF INTEREST}

The authors declare no conflict of interest.

\section{ACKNOWLEDGMENTS}

None

\section{AUTHOR CONTRIBUTIONS}

* Yanjun Ge and Danni Guo have equally contributed as first authors. DG and YG collected data and wrote the original draft. YG, XS, LZ and $\mathrm{RL}$ performed the clinical treatment. SP and $Y Z$ lead the writing and revised the manuscript.

\section{REFERENCE}

1. Warnakulasuriya S. Global epidemiology of oral and oropharyngeal cancer. Oral Oncol. 2009 Apr-May;45(4-5):309-316. doi: 10.1016/j.oraloncology.2008.06.002. Epub 2008 Sep 18. PMID: 18804401.

Full text links CrossRef PubMed Google Scholar Scopus WoS 2. Brown JS, Shaw RJ. Reconstruction of the maxilla and midface: introducing a new classification. Lancet Oncol. 2010 Oct;11(10):10011008. doi: 10.1016/S1470-2045(10)70113-3. PMID: 20932492. Full text links CrossRef PubMed Google Scholar Scopus WoS 3. Zhang DS, Zheng JW, Zhang CP, et al. [Multidisciplinary team model for patients with oral cancer and systemic diseases: an expert consensus]. Hua Xi Kou Qiang Yi Xue Za Zhi. 2020 Dec 1;38(6):603-615. Chinese. doi: 10.7518/hxkq.2020.06.001. PMID: 33377335; PMCID: PMC7738912.

Full text links PubMed Google Scholar Scopus WoS

4. Levine JP, Bae JS, Soares M, et al. Jaw in a day: total maxillofacial reconstruction using digital technology. Plast Reconstr Surg. 2013 Jun;131(6):1386-1391. doi: 10.1097/PRS.0b013e31828bd8d0. PMID: 23714799.

Full text links PubMed Google Scholar Scopus WoS

5. Tian T, Zhang T, Ma Q, et al. Reconstruction of mandible: a fully digital workflow from visualized iliac bone grafting to implant restoration. J Oral Maxillofac Surg. 2017 Jul;75(7):1403.e1-1403.e10. doi: 10.1016/j.joms.2017.02.022. Epub 2017 Mar 7. PMID: 28359016. Full text links CrossRef PubMed Google Scholar Scopus WoS 6. Zhang HQ, Li QX, Wang YY, et al. Combination of biomechanical evaluation and accurate placement of dental implants: a new concept of virtual surgery in maxillary and mandibular functional reconstruction. Br J Oral Maxillofac Surg. 2020 Jan;58(1):62-68. doi: 10.1016/j.bjoms.2019.10.310. Epub 2019 Nov 11. PMID: 31718915. Full text links PubMed Google Scholar Scopus WoS 7. Pan S, Guo D, Zhou Y, et al. Time efficiency and quality of outcomes in a model-free digital workflow using digital impression immediately after implant placement: a double-blind self-controlled clinical trial. Clin Oral Implants Res. 2019 Jul;30(7):617-626. doi: 10.1111/clr.13447. Epub 2019 May 13. PMID: 31021451.

Full text links CrossRef PubMed Google Scholar Scopus WoS 8. Liu X, Liu J, Feng H, Pan S. Accuracy of a milled digital implant surgical guide: an in vitro study. J Prosthet Dent. $2020 \mathrm{Dec} 10: 50022$ 3913(20)30654-5. doi: 10.1016/j.prosdent.2020.07.041. Epub ahead of print. PMID: 33309294

Full text links PubMed Google Scholar Scopus

9. Qiu SY, Kang YF, Ding MK, et al. Mandibular reconstruction with the iliac flap under the guidance of a series of digital surgical guides. J Craniofac Surg. 2021 Jul-Aug 01;32(5):1777-1779. doi: 10.1097/ SCS.0000000000007494. PMID: 33534315.

Full text links CrossRef PubMed Google Scholar Scopus WoS 10. Harbison RA, Shan XF, Douglas Z, et al. Navigation guidance during free flap mandibular reconstruction: a cadaveric trial. JAMA Otolaryngol Head Neck Surg. 2017 Mar 1;143(3):226-233. doi: 10.1001/ jamaoto.2016.3204. Erratum in: JAMA Otolaryngol Head Neck Surg. 2017 Mar 1;143(3):318. PMID: 27893003; PMCID: PMC8025841.

Full text links PubMed Google Scholar Scopus WoS

11. Nguyen TTH, Eo MY, Myoung H, et al. Implant-supported fixed and removable prostheses in the fibular mandible. Int J Implant Dent. 2020 Aug 11;6(1):44. doi: 10.1186/540729-020-00241-7. PMID: 32778982; PMCID: PMC7417466.

Full text links CrossRef PubMed Google ScholarWoS 
12. Kataoka T, Akagi Y, Kagawa C, et al. A case of effective oral rehabilitation after mandibular resection. Clin Case Rep. $2019 \mathrm{Sep}$ 27;7(11):2143-2148. doi: 10.1002/ccr3.2459. PMID: 31788267; PMCID: PMC6878091.

Full text links CrossRef PubMed Google Scholar Scopus WoS 13. de Souza LL, Pontes FSC, Fonseca FP, et al. Chondrosarcoma of the jaw bones: a review of 224 cases reported to date and an analysis of prognostic factors. Int J Oral Maxillofac Surg. 2019 Apr;48(4):452-460. doi: 10.1016/j.jom.2018.11.006. Epub 2018 Dec 6. PMID: 30528199.

Full text links CrossRef PubMed Google Scholar Scopus WoS

14. Karadwal A, Chatterjee S. Chondrosarcoma of maxilla. J Oral Maxillofac Pathol. 2018 Jan;22(Suppl 1):S35-S38. doi: 10.4103/jomfp. JOMFP_169_15.PMID: 29491602; PMCID: PMC5824514.

Full text links PubMed Google Scholar

15. Cuevas-Gonzalez JC, Reyes-Escalera JO, Gonzalez JL, et al. Primary maxillary chondrosarcoma: a case report. World J Clin Cases. 2020 Jan 6;8(1):126-132. doi: 10.12998/wjcc.v8.i1.126. PMID: 31970178; PMCID: PMC6962084.

Full text links PubMed Google Scholar Scopus WoS

16. De Cicco D, Colella G, Tartaro G, et al. Wide anterior maxillary reconstruction with equine bone xenograft: a case report of 24-month follow-up. Case Rep Surg. 2020 Oct 21;2020:8890935. doi: 10.1155/2020/8890935. PMID: 33145117; PMCID: PMC7596435. Full text links CrossRef PubMed Google Scholar WoS
17. Zhang WB, Peng X, Yu Y, et al. Expert consensus for the treatment algorithm for navigation assisted reconstruction of maxillofacial deformities. Chin J Dent Res. 2020;23(1):33-42. doi: 10.3290/j.cjdr.a44334. PMID: 32232227.

Full text links PubMed Google Scholar Scopus

18. Kang YF, Liang J, He Z, et al. Orbital floor symmetry after maxillectomy and orbital floor reconstruction with individual titanium mesh using computer-assisted navigation. J Plast Reconstr Aesthet Surg. 2020 Feb;73(2):337-343. doi: 10.1016/j. bjps.2019.07.014. Epub 2019 Aug 7. PMID: 31477492.

Full text links CrossRef PubMed Google Scholar Scopus WoS

19. Li R, Meng Z, Zhang Y, et al. Soft tissue management: a critical part of implant rehabilitation after vascularized free-flap reconstruction. J Oral Maxillofac Surg. 2021 Mar;79(3):560-574. doi: 10.1016/j.joms.2020.11.006. Epub 2020 Nov 14. PMID: 33279473.

Full text links PubMed Google Scholar Scopus WoS 20. Zhang L, Ding Q, Liu C, et al. Survival, function, and complications of oral implants placed in bone flaps in jaw rehabilitation: a systematic review. Int J Prosthodont. 2016 MarApr;29(2):115-25. doi: 10.11607/ijp.4402. PMID: 26929947. Full text links CrossRef PubMed Google Scholar Scopus WoS

Dr. Ge, DDS, has been a prosthodontist and lecturer at Peking University School and Hospital of Stomatology since 2009. As an ITI member, he carried out a large number of implant treatments using digital technology. He is good at and responsible for treating prosthetic patients, especially complex case treated with the multidisciplinary approach.

\section{0uestions}

\section{What is the average age range of male patients with maxillary chondrosarcoma?}
a. 40 to 55 ;
b. 35 to 45 ;
c. 25 to 36 ;
d. 65 to 75 .

\section{Which of the following options is not a method for maxillofacial surgery to reconstruct the maxilla?}

$\square$ a. Distraction osteogenesis;

b. Vascularized bone flap graft;

c. GBR;

d. Obturator.

\section{Which is the digital method commonly used in computer-assisted surgery?}

$\square$ a. Navigation surgery;

$\square$ b. Surgical guide plate;

$\square$ c. Virtual surgical planning;

$\square \mathrm{d}$. All of the above.

\section{What is the retention survival rate of implants on the bone flap (jaw reconstruction)?}
a. $78.5 \%$;
b. $93.2 \%$;
c. $96.8 \%$;
d. $89.7 \%$. 